\title{
Randomized, Double-Blinded, Multicenter, Placebo-Controlled Trial of Shenfu Injection for Treatment of Patients with Chronic Heart Failure during the Acute Phase of Symptom Aggravation (Yang and Qi Deficiency Syndrome)
}

\author{
Xianliang Wang, ${ }^{1}$ Zhiqiang Zhao, ${ }^{1}$ Jingyuan Mao $\mathbb{D}^{1},{ }^{1}$ Tinghai Du, ${ }^{2}$ Yuanping Chen, \\ Hao Xu $\left(\mathbb{D},{ }^{4}\right.$ Nan Liu, ${ }^{5}$ Xiaolong Wang, ${ }^{6}$ Jianguang Wu, ${ }^{7}$ Rong Li, ${ }^{5}$ Yong Xu, ${ }^{8}$ \\ Yingqiang Zhao, ${ }^{9}$ Lei Wang, ${ }^{10}$ Jingsong He, ${ }^{11}$ Junhua Zhang $\mathbb{D},{ }^{12}$ Jingbo Zhai, ${ }^{12}$ \\ Guoyuan Zhao, ${ }^{1}$ Yazhu Hou, ${ }^{1}$ Shuai Wang, ${ }^{1}$ and Chunxiang Liu $\mathbb{D}^{12}$ \\ ${ }^{1}$ First Teaching Hospital of Tianjin University of Traditional Chinese Medicine (TCM), Tianjin, 300193, China \\ ${ }^{2}$ The First Affiliated Hospital of Henan University of T.C.M., Henan, 450000, China \\ ${ }^{3}$ The First Affiliated Hospital of Guangxi University of Chinese Medicine, Guangxi, 530000, China \\ ${ }^{4}$ Xiyuan Hospital, China Academy of Chinese Medical Sciences (CACMS), Beijing, 100091, China \\ ${ }^{5}$ The First Affiliated Hospital of Guangzhou University of T.C.M, Guangzhou, 510450, China \\ ${ }^{6}$ Department of Cardiovascular, Shuguang Hospital Affiliated to Shanghai University of Traditional Chinese Medicine, \\ Shanghai, 201203, China \\ ${ }^{7}$ Affiliated Hospital of Jiangxi University of T.C.M., Jiangxi, 330000, China \\ ${ }^{8}$ Teaching Hospital of Chengdu University of T.C.M., Chengdu, 610000, China \\ ${ }^{9}$ Second Affiliated Hospital of Tianjin University of T.C.M., Tianjin, 300143, China \\ ${ }^{10}$ West China Hospital of Sichuan University, Sichuan, 610000, China \\ ${ }^{11}$ Ruikang Hospital Affiliated to Guangxi University of Chinese Medicine, Guangxi, 530000, China \\ ${ }^{12}$ Evidence-Based Medicine Center, Tianjin University of Traditional Chinese Medicine, Tianjin 300193, China
}

Correspondence should be addressed to Jingyuan Mao; jymao@126.com

Received 16 June 2018; Revised 2 January 2019; Accepted 20 January 2019; Published 25 February 2019

Academic Editor: Chang G. Son

Copyright (c) 2019 Xianliang Wang et al. This is an open access article distributed under the Creative Commons Attribution License, which permits unrestricted use, distribution, and reproduction in any medium, provided the original work is properly cited.

Background. Shenfu injection (SFI) has shown a remarkable therapeutic effect in patients with chronic heart failure (CHF) during the acute phase of symptom aggravation since it became commercially available in 1987 . However, the therapeutic effect of SFI has not been validated in a standard clinical study. As a pilot clinical trial, this study aimed to evaluate the safety and efficacy of SFI for treatment of CHF patients during the acute phase. Methods. A total of 160 patients experiencing acute phase CHF were enrolled in this study and randomly assigned to receive the placebo (placebo group, $150 \mathrm{ml}$ glucose (GS)) or SFI (SFI group, $50 \mathrm{ml} \mathrm{SFI}+100 \mathrm{ml} \mathrm{GS}$ ) in addition to their standard medications for CHF treatment. The treatment lasted for $7 \pm 1$ days, and the follow-up continued for $28 \pm 3$ days after treatment. The primary endpoints were New York Heart Association (NYHA) classification and Traditional Chinese Medicine (TCM) syndrome scores. Results. After $7 \pm 1$ days of treatment, the efficacy of SFI according to improvements in NYHA and TCM syndrome scores in the SFI group (78.38\% and $89.19 \%$, respectively) was significantly higher than that in the placebo group $(61.43 \%$ and $60.00 \%$, respectively; $\mathrm{P}<0.05)$. The SFI group had a longer increase in amplitude than the placebo group (113.00 m versus $82.99 \mathrm{~m}, \mathrm{P}<0.05)$. The incidence of adverse events and other safety indices showed no significant differences between the two groups. Conclusion. SFI combined with conventional therapy for treatment of CHF during acute symptom aggravation ameliorated the cardiac dysfunction and clinical symptoms and improved the patients' quality of life without any significant AEs compared with the conventional therapy alone. 


\section{Introduction}

Chronic heart failure $(\mathrm{CHF})$ is the leading cause of hospitalization in the aged population worldwide and negatively affects the quality of life of patients [1]. CHF patients commonly experience a vicious disease cycle: "hospitalization-improvement-discharge-rehospitalization.” Acute CHF is the most common type of acute heart failure, and, among patients with acute heart failure, the hospital mortality rate ranged from $4 \%$ to $11 \%$ [2-4], the vascular rehospitalization rate within 30 days was $7 \%-16 \%$ [5], and the mortality rate was $11.3 \%$ [6].

Based on the theory of Traditional Chinese Medicine (TCM), the major cause of heart failure is heart Yang deficiency that results from Qi inadequacy and blood stasis. Some traditional Chinese herbs have demonstrated safety and efficacy for the management of heart failure in either animal models or humans. For example, velvet antler of deer ameliorated cardiac dysfunction associated with heart failure in a rat myocardial model with heart failure following myocardial infarction [7], and Qili qiangxin capsules, another TCM, has been shown to be a beneficial auxiliary treatment for heart failure [8].

Shenfu injection (SFI) is a specific TCM extracted from two types of herbs, ginseng radix et rhizome, and radix aconiti carmichaeli. SFI has been shown to exhibit a variety of pharmacological activities, including elevating blood pressure and potentiating myocardial contractility, and has been used for many years to treat patients with cardiovascular diseases in China, such as dilated cardiomyopathy [9], heart failure [10], and acute myocardial infarction [11]. However, there have been few studies performed as a standard clinical trial to validate clinical observations of the safety and efficacy of SFI for treatment of heart diseases. In this pilot randomized multicenter, double-blinded placebo-controlled clinical trial, we aimed to assess the safety and efficacy of SFI for treatment of $\mathrm{CHF}$ during acute aggravation.

\section{Patients and Methods}

2.1. Study Protocol. This study was a randomized, doubleblinded, multicenter, placebo-controlled trial. A total of 160 patients at 11 clinical research centers in China were registered for this study (Supplemental Table 1). The study protocol was approved by the ethics committee of the First Teaching Hospital of Tianjin University of Traditional Chinese Medicine, and other ethics committees at the research centers that participated in this study. This pilot trial was published in Trials in May of 2015 [12]. All participants signed an informed consent form. This study was performed in compliance with the Declaration of Helsinki and has been registered in the Chinese Clinical Trial Register (http://www.chictr.org.cn; Register number: ChiCTR-TRC-12002857.

2.2. Inclusion and Exclusion Criteria. The inclusion criteria were as follows: (1) according to the diagnostic criteria defined for coronary heart disease, patients had occluded myocardial infarction with a stricture rate of the coronary artery main branch (at least 1 branch) of more than $50 \%$, regardless of percutaneous coronary intervention (PCI) or coronary artery bypass grafting (CABG) therapy; (2) according to the diagnostic criteria of $\mathrm{CHF}$ during acute aggravation, patients had a left ventricular ejection fraction (LVEF) $\leq 50 \%$ as determined by the improved Simpson method [13, 14] with one or more of the following: (i) stable cardiac function but sudden fatigue with no reason, (ii) difficulty breathing while doing physical work, or occasionally at night, and had to use a pillow to elevate the position of the head when sleeping, (iii) sudden serious expiratory dyspnea, orthopnoea, agitation, and/or fear; (iv) left ventricle being larger than before; basic heart rate being faster than before (+15-20 times/min); Traube's bruit, (dry) moist crackles, and wheezing rale in lungs especially in the bottom of lungs; (3) meeting the TCM criteria for yang and qi deficiency, which has the main symptoms of palpitations, shortness of breath (dyspnea), and fatigue; (4) age between 40 and 79 years; (5) New York Heart Association (NYHA) classification of III IV; and (6) signature on informed consent form.

The exclusion criteria were as follows: (1) acute coronary syndrome, acute myocardial infarction within 6 months, revascularization or intention to undergo revascularization within 6 months, cardiogenic shock, serious arrhythmias, cardiomyopathy, rheumatic valvular heart disease, myocarditis, constrictive pericarditis, and pulmonary embolism within a week; (2) severe liver and renal insufficiency (glutamic oxalacetic transaminase [ALT] $\geq 3$ times normal upper limit, creatinine $[\mathrm{Cr}] \geq 3 \mathrm{mg} / \mathrm{dL}$ ); (3) severe primary disease in the endocrine and hematopoietic system; (4) intention to become pregnant or breastfeed; (5) psychopath; (6) allergy to SFI; (7) participation in other trial within the previous 3 months; (8) life expectancy $<3$ months as judged by the investigator; and (9) failure to fulfill or obey the study guidelines as judged by the investigator.

2.3. Interventions. After application of the inclusion and exclusion criteria, a total of 160 patients were finally enrolled in our study and randomly assigned to two groups: the placebo group, in which 80 patients were given the placebo (GS $150 \mathrm{ml}$ ), and the SFI group, in which 80 patients were treated with SFI (SFI $50 \mathrm{ml}+$ GS $100 \mathrm{ml}$ ). Patients in both groups simultaneously received their usual care and medications such as diuretics, angiotensin converting enzyme inhibitors (ACEIs), or angiotensin II receptor blockers (ARBs), $\beta$-receptor blocker, aldosterone-receptor blocker, digoxin, and vasodilator substance prescribed for $\mathrm{CHF}$ by the attending physicians. The study medication was uniformly labeled by the sponsor, blinded by the statistics unit, and then sent to each center. Special drug administrators were established in each subcenter, and the researchers informed the nurse after random access to the drug number. The nurse was responsible for preparing the medicine, confecting solution, and serving the transfusion to patients with a disposable optical infusion device. Therefore, the study was not blinded to the nurse but to the researchers, patients, and statisticians. The participants were given SFI or placebo once a day during the $7( \pm 1)$-day course of treatment. Each patient's health status was evaluated before and after treatment including curative indices and safety indices, and all patients were followed 
up for $28 \pm 3$ days after administration of the medication by telephone or outpatient visit. The follow-up information included cardiovascular events and rehospitalization (Supplemental Figure 1).

2.4. Endpoints. The primary endpoints were NYHA classification and TCM syndrome scores. The secondary endpoints included Lee's CHF scores, 6-minute walk distance (6MWD), LVEF, and the incidence rate of cardiovascular events and heart failure emergency/rehospitalization. The safety endpoints included tests of blood, urine, stool, the levels of serum $\mathrm{K}, \mathrm{Na}, \mathrm{Cl}, \mathrm{ALT}$, aspartate aminotransferase (AST), blood urea nitrogen (BUN), and $\mathrm{Cr}$, and the occurrence of adverse events (AEs)/adverse drug reactions (ADRs).

2.5. Efficiency Standard. The efficiency standard was formulated in reference to "the principle of clinical research on treating heart failure with new Chinese Medicine [15]" (2002).

\section{Heart Function Efficiency Standard}

Excellent: heart failure was essentially ameliorated or the NYHA classification increased by at least 2 levels.

Valid: NYHA classification increased by 1 level.

Invalid: NYHA classification remained the same before and after the treatment.

Worsened: NYHA classification decreased by at least 1 level.

\section{Efficiency Standard for Yang and Qi Deficiency Syndrome}

Excellent: Clinical symptoms were markedly improved, and the TCM syndrome score decreased by $70 \%$ compared with that before treatment.

Valid: Clinical symptoms were improved, $30 \% \leq$ TCM syndrome score $<70 \%$.

Invalid: Clinical symptoms were not improved.

Worsened: Clinical symptoms were worsened. The TCM syndrome score increased.

\section{Lee’s Heart Failure Score}

Excellent: Scores decreased by $75 \%$ compared with that before treatment.

Valid: $50 \% \leq$ decreased scores $<75 \%$.

Invalid: $0 \leq$ decreased scores $<50 \%$.

Worsened: Decreased scores $<0$.

Sample Size Calculation. This study was a pilot clinical trial. The sample size was calculated based on a $20 \%$ expulsion rate in a clinical study and an expert's opinion. Hence, we recruited 160 patients to this study, who were subsequently allocated at a 1:1 ratio to the SFI and placebo groups.

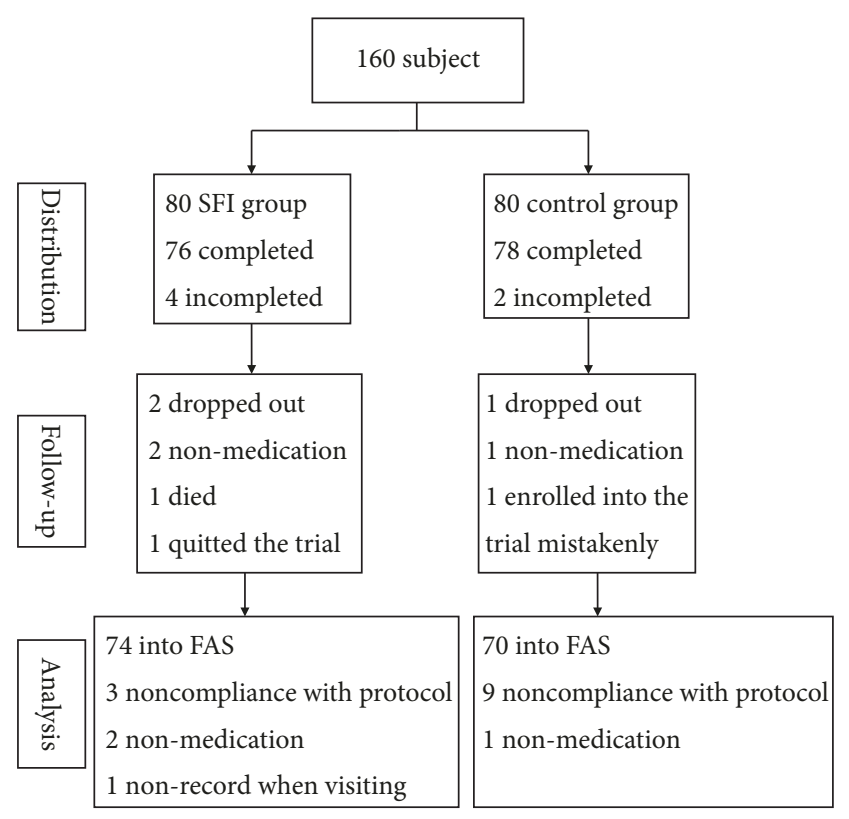

Figure 1: Flow chart of patient selection and study design.

2.6. Statistical Analysis. All statistical analyses were performed at an independent institute-Tianjin Institute of Traditional Chinese Medicine Engineering-with SAS software, version 9.1.3. Data were analyzed according to the full analysis set principle. Continuous variables are presented as the mean \pm standard deviation (SD). The comparability of the characteristics between the two study groups was assessed using a two-sample Student t-test for continuous variables and the chi-square test or Wilcoxon test, when appropriate, for categorical variables. The Wilcoxon paired signed-rank test was used for within-group comparisons. The statistical difference in the $\mathrm{AE}$ incidence rate between the two study groups was assessed using $\chi^{2}$ test (or Fisher exact test if not appropriate). A P value $<0.05$ was considered statistically significant, and all tests were two-tailed.

\section{Results}

3.1. Study Population. From April 18, 2013, to August 2, 2015, a total of 160 patients were enrolled in this study and randomly assigned to the placebo and SFI groups at a 1:1 ratio. Among these participants, 154 were followed up for $28 \pm 3$ days after the treatment (76 in the SFI group and 78 in the placebo group); 144 were enrolled in the full analysis set (FAS) (74 in the SFI group and 70 in the placebo group); and 137 were enrolled in the per-protocol set (PPS) (71 in the SFI group and 66 in the placebo group). Medication compliance of all patients was recorded (102.32\% in the SFI group and $101.22 \%$ in placebo group; Figure 1).

3.2. Comparison of Demographic and Baseline Clinical Characteristics of Participants between the SFI and Placebo Groups. The recorded demographic and clinical characteristics included age, gender, medical history, course of heart 
TABLE 1: Comparison of demographic and baseline clinical characteristics of participants between the SFI and placebo groups.

\begin{tabular}{|c|c|c|c|c|}
\hline Characteristic & SFI & Placebo & Statistics & $\mathrm{P}$ value \\
\hline \multicolumn{5}{|l|}{ Basic information } \\
\hline Age $(\bar{x} \pm s$, yrs $)$ & $68.58 \pm 8.42$ & $68.14 \pm 8.73$ & -0.3042 & 0.7610 \\
\hline \multicolumn{5}{|l|}{ Gender } \\
\hline Male $\mathrm{n}(\%)$ & $42(56.76 \%)$ & $48(68.57 \%)$ & 2.1424 & 0.1433 \\
\hline Female $\mathrm{n}(\%)$ & $32(43.24 \%)$ & $22(31.43 \%)$ & & \\
\hline Weight $(\bar{x} \pm s, \mathrm{~kg})$ & $65.43 \pm 13.10$ & $66.26 \pm 11.82$ & 0.5744 & 0.5657 \\
\hline Height $(\bar{x} \pm s, \mathrm{~cm})$ & $164.10 \pm 7.30$ & $166.00 \pm 6.60$ & 1.63 & 0.1052 \\
\hline $\operatorname{SBP}(\bar{x} \pm s, \mathrm{mmHg})$ & $132.55 \pm 21.89$ & $133.27 \pm 19.47$ & 0.2648 & 0.7912 \\
\hline $\operatorname{DBP}(\bar{x} \pm s, \mathrm{mmHg})$ & $77.26 \pm 10.77$ & $77.64 \pm 11.04$ & 0.3134 & 0.7540 \\
\hline Heart rate $(\bar{x} \pm s$, bpm $)$ & $86.5 \pm 21.6^{\star}$ & $76.1 \pm 20.0$ & -3.1526 & 0.0016 \\
\hline \multicolumn{5}{|l|}{ Medical history } \\
\hline Disease course of CHF $(\bar{x} \pm s, \mathrm{yrs})$ & $4.62 \pm 6.07$ & $3.76 \pm 3.69$ & -0.4280 & 0.6687 \\
\hline Disease course of acute exacerbation $(\bar{x} \pm s$, days $)$ & $6.08 \pm 5.07$ & $6.94 \pm 8.27$ & 0.1853 & 0.8530 \\
\hline History of myocardial infarction $\mathrm{n}(\%)$ & $38(52.05 \%)$ & $43(61.43 \%)$ & 1.2785 & 0.2582 \\
\hline History of arrhythmia n(\%) & $20(27.03 \%)$ & $19(27.14 \%)$ & 0.0002 & 0.9875 \\
\hline History of hypertension $\mathrm{n}(\%)$ & $51(70.83 \%)$ & $44(62.86 \%)$ & 1.0197 & 0.3126 \\
\hline History of diabetes $\mathrm{n}(\%)$ & $26(35.14 \%)$ & $16(22.86 \%)$ & 2.6248 & 0.1052 \\
\hline \multicolumn{5}{|l|}{ Medication use } \\
\hline Antiplatelet $\mathrm{n}(\%)$ & $63(85.14 \%)$ & $61(87.14 \%)$ & 0.1212 & 0.7277 \\
\hline Beta-blockers n(\%) & $36(48.65 \%)$ & $44(62.86 \%)$ & 2.9412 & 0.0863 \\
\hline ACE inhibitors $\mathrm{n}(\%)$ & $32(43.24 \%)$ & $35(50.00 \%)$ & 0.6601 & 0.4165 \\
\hline ARB inhibitors $\mathrm{n}(\%)$ & $23(31.08 \%)$ & $19(27.14 \%)$ & 0.2700 & 0.6033 \\
\hline Statins $\mathrm{n}(\%)$ & $57(77.03 \%)$ & $50(71.43 \%)$ & 0.5905 & 0.4422 \\
\hline Nitric acid lipid n(\%) & $44(59.46 \%)$ & $44(62.86 \%)$ & 0.1747 & 0.6759 \\
\hline Ca-antagonist $\mathrm{n}(\%)$ & $18(24.32 \%)$ & $17(24.29 \%)$ & 0.0000 & 0.9957 \\
\hline Aldosterone receptor antagonist $\mathrm{n}(\%)$ & $50(67.57 \%)$ & $43(61.43 \%)$ & 0.5927 & 0.4414 \\
\hline Diuretics n(\%) & $45(60.81 \%)$ & $35(50.00 \%)$ & 1.7027 & 0.1919 \\
\hline Digoxin $n(\%)$ & $29(39.19 \%)$ & $24(34.29 \%)$ & 0.3719 & 0.5420 \\
\hline \multicolumn{5}{|l|}{ Clinical index } \\
\hline TCM syndrome score $(\bar{x} \pm s)$ & $24.54 \pm 7.90$ & $23.37 \pm 7.13$ & -1.1902 & 0.2340 \\
\hline Lee's heart failure $(\bar{x} \pm s)$ & $6.36 \pm 3.25$ & $5.71 \pm 2.92$ & -1.1787 & 0.2385 \\
\hline $6 \mathrm{MWD}(\bar{x} \pm s, \mathrm{~m})$ & $163.28 \pm 153.48$ & $185.70 \pm 143.30$ & 1.0504 & 0.2935 \\
\hline $\operatorname{LVEF}(\bar{x} \pm s, \%)$ & $38.69 \pm 8.52$ & $39.82 \pm 8.04$ & 0.7183 & 0.4726 \\
\hline \multicolumn{5}{|l|}{ NYHA classification } \\
\hline III $\mathrm{n}(\%)$ & $46(62.16 \%)$ & $51(72.86 \%)$ & 1.8715 & 0.1713 \\
\hline IV n $(\%)$ & $28(37.84 \%)$ & $19(27.14 \%)$ & & \\
\hline
\end{tabular}

${ }^{\star} \mathrm{P}<0.01$ compared to control group

failure in the acute aggravation phase, information of background medication, NYHA classification, TCM syndrome score, Lee's heart failure score, 6MWD, and LVEF. The distributions of the demographic and clinical characteristics between the SFI and placebo groups were well balanced and homogeneous $(\mathrm{P}>0.05$. Table 1$)$, except that the average heart rate in the SFI group was significantly higher than that in placebo group $(\mathrm{P}<0.05$, Table 1$)$.

3.3. Comparison of Primary Endpoints between SFI and Placebo Group. SFI treatment significantly improved the NYHA classification by $78.38 \%$ compared to the $61.43 \%$ increase observed in the placebo group $(\mathrm{P}=0.0026$, relative risk $[R R]=1.2759,95 \%$ confidence interval $[\mathrm{CI}]$ :
1.0231-1.5913; Table 2). Similarly, SFI treatment exhibited a high efficacy for improving the TCM syndrome score (by $89.19 \%$ ) compared to the $60.00 \%$ increase observed in the placebo group $(\mathrm{P}<0.001, \mathrm{RR}=1.4865,95 \% \mathrm{CI}$ : $1.2085-1.8285$; Table 3).

3.4. Comparison of the Secondary Endpoints between the SFI and Placebo Groups. SFI treatment had a significantly higher effective rate (70.27\%) for improving Lee's heart failure score compared with placebo treatment $(52.17 \% ; \mathrm{P}=0.0262$, $\mathrm{RR}=1.3468$, 95\%CI: 1.0280-1.7646; Table 4). Also, SFI treatment greatly increased the 6MWD $(113.00 \mathrm{~m})$ compared with that in the placebo group ( $82.99 \mathrm{~m}, \mathrm{P}=0.0281$; Table 5). Although patients in the SFI group tended to have better 
TABLE 2: Comparison of NYHA classification between SFI and placebo group.

\begin{tabular}{|c|c|c|c|c|c|c|c|}
\hline & Excellent & Valid & Invalid & Worsened & Effective rate & Statistics & $\mathrm{P}$ value \\
\hline SFI $(n=74)$ & 18 & 40 & 16 & 0 & $78.38 \%$ & \multirow{2}{*}{4.9344} & \multirow{2}{*}{0.026} \\
\hline Placebo $(n=70)$ & 8 & 35 & 27 & 0 & $61.43 \%$ & & \\
\hline
\end{tabular}

Effective rate was defined as proportion of all patients who experienced an excellent or valid outcome. Similarly, the ineffective rate was defined as the proportion of all patients who experienced an invalid and worsened outcome.

TABLE 3: Comparison of improvement in TCM syndrome score between SFI and placebo groups.

\begin{tabular}{|c|c|c|c|c|c|c|c|}
\hline & Excellent & Valid & Invalid & Worsened & Effective rate & Statistics & $\mathrm{P}$ value \\
\hline SFI $(n=74)$ & 22 & 44 & 7 & 1 & $89.19 \%$ & \multirow{2}{*}{16.3459} & \multirow{2}{*}{$<0.001$} \\
\hline Control $(n=70)$ & 14 & 28 & 28 & 0 & $60.00 \%$ & & \\
\hline
\end{tabular}

Effective rate was defined as proportion of all patients who experienced an excellent or valid outcome. Similarly, the ineffective rate was defined as the proportion of all patients who experienced an invalid and worsened outcome.

TABLE 4: Comparison of Lee's heart failure score between SFI and placebo groups.

\begin{tabular}{|c|c|c|c|c|c|c|c|}
\hline & Excellent & Valid & Invalid & Worsened & Effective rate & Statistics & $P$ value \\
\hline SFI $(n=74)$ & 23 & 29 & 21 & 1 & $70.27 \%$ & \multirow{2}{*}{4.9403} & \multirow{2}{*}{0.0262} \\
\hline Placebo $(n=69)$ & 15 & 21 & 33 & 0 & $52.17 \%$ & & \\
\hline
\end{tabular}

Effective rate was defined as proportion of all patients who experienced an excellent or valid outcome. Similarly, the ineffective rate was defined as the proportion of all patients who experienced an invalid and worsened outcome.

TABLE 5: Comparison of change in 6MWD (pre-to-post treatment) between SFI and placebo groups.

\begin{tabular}{|c|c|c|c|c|}
\hline Endpoint & & SFI $(n=70)$ & Placebo $(n=69)$ & P value \\
\hline $6 \mathrm{MWD}$ & Posttreatment-pretreatment & $113.00 \pm 117.55$ & $82.99 \pm 127.99$ & 0.0281 \\
\hline
\end{tabular}

TABLE 6: Comparison of change in LVEF (pre-to-post treatment) between SFI and placebo groups.

\begin{tabular}{|c|c|c|c|c|}
\hline Endpoint & & SFI $(n=72)$ & Placebo $(n=67)$ & P value \\
\hline LVEF & Post-treatment - pre-treatment & $6.58 \pm 9.11$ & $4.53 \pm 8.21$ & 0.2347 \\
\hline
\end{tabular}

LVEF than those in the placebo group, no statistical difference was observed $(\mathrm{P}>0.05$; Table 6$)$. No composite cardiac events (CCEs) or death occurred in the SFI group, whereas 2 CCEs and 1 death occurred in the placebo group. However, no statistical differences in these endpoints were observed between the two groups ( $\mathrm{P}>0.05$; Table 7$)$.

3.5. Safety Evaluation for SFI. No statistical differences in the occurrence of AEs were observed between the SFI and placebo groups (Table 8). Only 2 ADRs occurred in the SFI group, and the difference from the placebo group was not significant $(\mathrm{P}>0.05$; Table 9$)$. In addition, there were no differences in the laboratory indexes between the SFI and placebo groups $(\mathrm{P}>0.05$; Table 10$)$.

\section{Discussion}

SFI, as a form of TCM, has been long used in clinical practice in China to treat $\mathrm{CHF}$ and has achieved favorable outcomes. However, to the best of our knowledge, this study was the first standard clinical trial, i.e., randomized, multicenter, double-blinded, placebo-controlled trial, to explore the safety and efficacy of SFI for treatment of patients in the acute phase of CHF who were simultaneously receiving standard
TABLE 7: Comparison of CCEs between SFI and placebo groups.

\begin{tabular}{lcc}
\hline Endpoint & SFI $(\mathrm{n}=74)$ & Placebo $(\mathrm{n}=70)$ \\
\hline CCE & $0(0.00 \%)$ & $2(2.86 \%)$ \\
$\quad$ revascularization & $0(0.00 \%)$ & $2(2.86 \%)$ \\
Death incident & $0(0.00 \%)$ & $1(1.43 \%)$ \\
\hline
\end{tabular}

treatments. The major findings included the following: (1) SFI significantly improved the clinical symptoms of these patients; (2) SFI significantly improved cardiac tolerance; and (3) SFI did not induce AEs or ADRs.

With the acceleration of the aging population and advancement in the treatment of human diseases including cardiovascular disorders, the human life expectancy has been extended. However, the number of patients with $\mathrm{CHF}$ has also been increasing. Patients with $\mathrm{CHF}$ are often hospitalized with acute symptom exacerbation due to various causes. Such patients mainly present with deterioration of cardiac function accompanied by a decline in athletic tolerance. The quest for treatments to alleviate symptoms of CHF during the acute phase of symptom exacerbation has been an active area of research in the clinic. TCM has been used clinically to treat 
TABLE 8: Comparison of AEs (top 10) between SFI and placebo groups.

\begin{tabular}{lccc}
\hline ADE & SFI $(\mathrm{n}=78)$ & Placebo $(\mathrm{n}=79)$ & All $(\mathrm{n}=157)$ \\
\hline Renal dysfunction & $6(7.69 \%)$ & $4(5.06 \%)$ & $10(6.37 \%)$ \\
Liver dysfunction & $1(1.28 \%)$ & $2(2.53 \%)$ & $3(1.91 \%)$ \\
Urinary system infection & $5(6.41 \%)$ & $3(3.80 \%)$ & $8(5.10 \%)$ \\
Urine protein & $1(1.28 \%)$ & $1(1.27 \%)$ & $2(1.27 \%)$ \\
Pulmonary infection & $1(1.28 \%)$ & $0(0.00 \%)$ & $1(0.64 \%)$ \\
Anemia & $1(1.28 \%)$ & $0(0.00 \%)$ & $1(0.64 \%)$ \\
Hypoglycemia & $1(1.28 \%)$ & $0(0.00 \%)$ & $1(0.64 \%)$ \\
Chills & $1(1.28 \%)$ & $0(0.00 \%)$ & $1(0.64 \%)$ \\
Erythra & $1(1.28 \%)$ & $0(0.00 \%)$ & $1(0.64 \%)$ \\
Diarrhea & $0(0.00 \%)$ & $1(1.27 \%)$ & $1(0.64 \%)$ \\
Ureteral calculi cut into stone & $1(1.28 \%)$ & $0(0.00 \%)$ & $1(0.64 \%)$ \\
\hline
\end{tabular}

TABLE 9

(a) Comparison of ADRs (top 10) between SFI and placebo groups

\begin{tabular}{lccc}
\hline ADR & SFI $(\mathrm{n}=78)$ & Placebo $(\mathrm{n}=79)$ & All $(\mathrm{n}=157)$ \\
\hline Chills & $1(1.28 \%)$ & $0(0.00 \%)$ & $1(0.64 \%)$ \\
Erythra & $1(1.28 \%)$ & $0(0.00 \%)$ & $1(0.64 \%)$ \\
Total & $2(2.56 \%)$ & $0(0.00 \%)$ & $2(1.27 \%)$ \\
\hline
\end{tabular}

(b) Accessory: list of ADRs

\begin{tabular}{lccc}
\hline Subject number & Group & $\begin{array}{c}\text { ADR } \\
\text { symptom }\end{array}$ & ADR description \\
\hline 006002 & SFI & Chill & $\begin{array}{c}\text { Chill occurred after injection of 10 ml SFI. } \\
\text { The subject felt itchy skin after } 1 \text { day of } \\
\text { medication. Skin flushing was seen on the } \\
\text { upper parts of the chest and abdomen, } \\
\text { the medial buttocks, and both lower } \\
\text { limbs. There was scratch, but no break. }\end{array}$ \\
\hline
\end{tabular}

CHF patients with acute symptom exacerbation in China for a long time [16-18], suggesting that TCM may serve as a complementary therapy in combination with standard treatments. In the present study, we evaluated the efficacy of SFI, an old TCM prepared with a new method, for the treatment of $\mathrm{CHF}$ patients during the acute exacerbation phase. We found that patients in the SFI treatment group exhibited significantly improved primary endpoints such as NYHA classification and TCM syndrome scores. Mechanistically, the therapeutic effects of SFI may be attributed to its coronary arterial dilation via mediation of NO synthesis/release [19], apoptosis suppression via regulating Blc2 activity [20], and activation of myocardial cell endothelial nitric oxide synthase (eNOS) via targeting PI3K/Akt pathway [21].

NYHA, as a simple, accurate index for evaluating heart function, can reflect the severity of acute heart failure, which has been shown to be closely related to survival $[22,23]$. The TCM syndrome score system, which is based on TCM symptoms and signs, is one of the most important and most commonly used indexes for evaluating the effectiveness of a TCM in the treatment of a disease [24]. As a pilot trial, this study used both the NYHA classification and TCM syndrome score as the primary endpoints to examine the efficacy of SFI for treatment of CHF patients. The results showed that
SFI treatment in combination with other standard therapies greatly benefited cardiac functional classification and TCM syndrome score compared with the improvements seen in the placebo group, which coincided with improved Lee's heart failure scores and 6MWDs. These findings suggest that SFI treatment may benefit patients in the acute phase of CHF. Notably, no statistical difference in LVEF was observed between the SFI and placebo group, indicating that the improvement of heart functional classification and clinical symptoms by SFI might not be necessarily related to an improvement in cardiac pumping capability. In addition, SFI treatment tended to improve CCEs, but no significant difference was detected between the two groups, which can probably be attributed to the small sample size of our study.

In the present study, we also evaluated the safety of SFI and found no significant differences in the occurrence of ADRs and AEs between the SFI and placebo group, suggesting the appreciable safety of SFI in clinical use.

\section{Conclusion}

In the present study, we demonstrated that SFI treatment in combination with conventional therapy for CHF in the acute phase ameliorated cardiac dysfunction and clinical 
TABLE 10: Comparison of incidence of abnormal laboratory indexes between SFI and placebo groups.

\begin{tabular}{|c|c|c|}
\hline Laboratory index & SFI $(n=78)$ & Placebo $(n=79)$ \\
\hline \multicolumn{3}{|l|}{ Blood routine } \\
\hline White blood cell count & $12(16.00 \%)$ & $9(11.84 \%)$ \\
\hline Red blood cell count & $10(13.33 \%)$ & $9(11.84 \%)$ \\
\hline Hemoglobin & $15(20.00 \%)$ & $12(15.79 \%)$ \\
\hline Blood platelet count & $11(14.67 \%)$ & $8(10.53 \%)$ \\
\hline \multicolumn{3}{|l|}{ Urine routine } \\
\hline Urine protein & $6(11.11 \%)$ & $10(16.13 \%)$ \\
\hline Urine sugar & $4(7.41 \%)$ & $3(4.84 \%)$ \\
\hline Urine erythrocyte & $8(14.81 \%)$ & $10(16.13 \%)$ \\
\hline Urine white blood cell count & $11(20.37 \%)$ & $8(12.90 \%)$ \\
\hline \multicolumn{3}{|l|}{ Stool routine } \\
\hline Red blood cell & $0(0.00 \%)$ & $0(0.00 \%)$ \\
\hline White blood cell & $2(7.14 \%)$ & $0(0.00 \%)$ \\
\hline Occult blood & $6(21.43 \%)$ & $2(5.13 \%)$ \\
\hline \multicolumn{3}{|l|}{ Hepatic and renal function } \\
\hline Alanine transaminase (ALT) & $6(8.45 \%)$ & $6(8.57 \%)$ \\
\hline Glutamic oxalacetic transaminase (AST) & $7(9.59 \%)$ & $5(6.76 \%)$ \\
\hline Total bilirubin & $2(2.90 \%)$ & $5(7.35 \%)$ \\
\hline Urea nitrogen & 17 (22.97\%) & $13(17.33 \%)$ \\
\hline Creatinine & $16(21.33 \%)$ & $20(26.67 \%)$ \\
\hline \multicolumn{3}{|l|}{ Electrolytes } \\
\hline K & $5(6.58 \%)$ & $2(2.60 \%)$ \\
\hline $\mathrm{Na}$ & $5(6.58 \%)$ & $6(7.79 \%)$ \\
\hline $\mathrm{Cl}$ & $3(3.95 \%)$ & $5(6.49 \%)$ \\
\hline
\end{tabular}

symptoms, increased the 6MWD, and improved patients' quality of life compared with the conventional therapy alone. We also demonstrated the safety of SFI in clinical use. Future studies with a large cohort are needed to further corroborate our findings and conclusions.

\section{Limitations}

This study was conducted only in some areas, and the observation period was short. Also, we did not see the statistical differences in some endpoint indicators such as CCEs or deaths between these groups. In the future, the study of endpoint events can be carried out sufficiently with greater research funds and manpower.

\section{Abbreviations}

SFI: Shenfu injection

CHF: Chronic heart failure

TCM: Traditional Chinese Medicine

AE: Adverse event

ADR: Adverse drug reaction

NYHA: New York Heart Association

CABG: Coronary artery bypass grafting

PCI: Percutaneous coronary intervention

6MWD: 6-min walking distance

AST: Aspartate aminotransferase
BUN: Blood urea nitrogen

FAS: Full analysis set

PPS: Per-protocol set

GS: Glucose.

\section{Data Availability}

The datasets used and analyzed during the current study are available from the corresponding author on reasonable request.

\section{Conflicts of Interest}

The authors declare that they have no conflicts of interest

\section{Authors' Contributions}

Jingyuan Mao conceived and designed the experiments. Xianliang Wang, Zhiqiang Zhao, Tinghai Du, Yuanping Chen, Hao Xu, Nan Liu, Xiaolong Wang, Jianguang $\mathrm{Wu}$, Rong Li, Yong Xu, Yingqiang Zhao, Lei Wang, Jingsong He, Guoyuan Zhao, Yazhu Hou, and Shuai Wang performed the experiments. Junhua Zhang and Chunxiang Liu randomized and managed the data. Jingbo Zhai analyzed the data. Xianliang Wang and Zhiqiang Zhao wrote the paper. Xianliang Wang and Zhiqiang Zhao contributed equally. 


\section{Acknowledgments}

(1) Tianjin science and technology project: clinical medicine research center of Internal medicine of TCM in Tianjin (15ZXLCSY00020) and (2) "Innovation team development plan" of Ministry of Education-Research on the prevention and treatment of cardiovascular diseases in traditional Chinese medicine (IRT_16R54) are acknowledged.

\section{Supplementary Materials}

Supplemental Table 1: List of participating institutions. Supplemental Table 2: CONSORT 2010 checklist of information to include when reporting a randomised trial. Supplemental Figure 1: Trial flow chart. (Supplementary Materials)

\section{References}

[1] N. Azad and G. Lemay, "Management of chronic heart failure in the older population," Journal of Geriatric Cardiology, vol. 11, no. 4, pp. 329-337, 2014.

[2] K. F. Adams Jr., G. C. Fonarow, C. L. Emerman et al., "Characteristics and outcomes of patients hospitalized for heart failure in the United States: rationale, design, and preliminary observations from the first 100,000 cases in the Acute Decompensated Heart Failure National Registry (ADHERE)," American Heart Journal, vol. 149, no. 2, pp. 209-216, 2005.

[3] M. S. Nieminen, D. Brutsaert, K. Dickstein et al., "EuroHeart Failure Survey II (EHFS II): a survey on hospitalized acute heart failure patients: description of population," European Heart Journal, vol. 27, no. 22, pp. 2725-2736, 2006.

[4] A. Rudiger, V.-P. Harjola, A. Müller et al., "Acute heart failure: Clinical presentation, one-year mortality and prognostic factors," European Journal of Heart Failure, vol. 7, no. 4, pp. 662670, 2005.

[5] J. A. Ezekowitz, C. Van Walraven, F. A. McAlister, P. W. Armstrong, and P. Kaul, "Impact of specialist follow-up in outpatients with congestive heart failure," Canadian Medical Association Journal, vol. 172, no. 2, pp. 189-194, 2005.

[6] G. C. Fonarow, "Epidemiology and risk stratification in acute heart failure," American Heart Journal, vol. 155, no. 2, pp. 200207, 2008.

[7] M.-J. Shao, S.-R. Wang, M.-J. Zhao et al., “The effects of velvet antler of deer on cardiac functions of rats with heart failure following myocardial infarction," Evidence-Based Complementary and Alternative Medicine, vol. 2012, Article ID 825056, 5 pages, 2012.

[8] X. Li, J. Zhang, and J. Huang, "A multicenter, randomized, double-blind, parallel-group, placebo-controlled study of the effects of qili qiangxin capsules in patients with chronic heart failure," Journal of the American College of Cardiology, vol. 62, no. 12, pp. 1065-1072, 2013.

[9] Y. Nie, H. Song, and C. Qi, "Effect of Shenfu injection in the treatment of dilated cardiomyopathy with heart failure clinical observation of 38 cases," China Medicine and Pharmacy, vol. 2, pp. 64-64, 2012.

[10] S. Wen-Ting, C. Fa-Feng, X. Li et al., "Chinese medicine shenfu injection for heart failure: a systematic review and meta-analysis," Evidence-Based Complementary and Alternative Medicine, vol. 2012, Article ID 713149, 25 pages, 2012.

[11] Y. Jin, H. Gao, X. Zhang, H. Ai, X. Zhu, and J. Wang, "Shenfu Injection inhibits inflammation in patients with acute myocardial infarction complicated by cardiac shock," Chinese Journal of Integrative Medicine, vol. 23, no. 3, pp. 170-175, 2017.

[12] C. Liu, Y. Hou, X. Wang et al., "Clinical assessment of Shenfu injection loading in the treatment of patients with exacerbation of chronic heart failure due to coronary heart disease: study protocol for a randomized controlled trial," Trials, vol. 16, no. 1, article 222, 2015.

[13] R. S. Vasan, M. G. Larson, E. J. Benjamin, J. C. Evans, C. K. Reiss, and D. Levy, "Congestive heart failure in subjects with normal versus reduced left ventricular ejection fraction: Prevalence and mortality in a population-based cohort," Journal of the American College of Cardiology, vol. 33, no. 7, pp. 19481955, 1999.

[14] W. J. Paulus, F. A. Flachskampf, O. A. Smiseth, and A. G. Fraser, "How to diagnose diastolic heart failure: a consensus statement on the diagnosis of heart failure with normal left ventricular ejection fraction by the Heart Failure and Echocardiography Associations of the European Society of Cardiology: reply," European Heart Journal, vol. 28, no. 20, pp. 2686-2687, 2007.

[15] X. Y. Zheng, Guidelines on The Clinical Research of New Chinese Medicine Drugs, Chinese Pharmaceutical Technology Press, 2002.

[16] L. Zhu, Q. H. Chen, and W. Xu, "Clinical observation of 40 cases of acute heart failure with the therapy of SFI," Chengdu University of Traditional Chinese Medicine, vol. 9, pp. 190-192, 2016.

[17] S. H. Liu, J. L. Chen, and M. F. He, "Clinical observation of acute left heart failure with the therapy of SFI," China TCM Emergency, vol. 8, pp. 1339-1341, 2015.

[18] B. X. Xie, X. F. Huang, and W. S. Li, "Clinical observation of acute heart failure with the therapy of SFI," China TCM Emergency, vol. 5, pp. 935-936, 2014.

[19] Y. H. Li, B. Yu, Z. Z. Duan et al., "The coronary dilation effect of Shen Fu injection was mediated through NO," PLoS ONE, vol. 9, no. 3, Article ID e92415, 2014.

[20] Y.-L. Wang, C.-Y. Wang, B.-J. Zhang, and Z.-Z. Zhang, "Shenfu injection suppresses apoptosis by regulation of $\mathrm{Bcl}-2$ and caspase-3 during hypoxia/reoxygenation in neonatal rat cardiomyocytes in vitro," Molecular Biology Reports, vol. 36, no. 2, pp. 365-370, 2009.

[21] S.-Y. Zheng, J. Sun, X. Zhao, and J.-G. Xu, "Protective effect of Shen-Fu on myocardial ischemia-reperfusion injury in rats," American Journal of Chinese Medicine, vol. 32, no. 2, pp. 209220, 2004.

[22] S. M. Dunlay, M. M. Redfield, S. A. Weston et al., "Hospitalizations after heart failure diagnosis. A community perspective," Journal of the American College of Cardiology, vol. 54, no. 18, pp. 1695-1702, 2009.

[23] S. A. Hunt, W. T. Abraham, and M. H. Chin, "ACC/AHA 2005 guideline update for the diagnosis and management of chronic heart failure in the adult: a report of the American College of Cardiology/American Heart Association Task Force on Practice Guidelines (Writing Committee to Update the 2001 Guidelines for the Evaluation and Management of Heart Failure): developed in collaboration with the American College of Chest Physicians and the International Society for heart and lung transplantation: endorsed by the Heart Rhythm Society," Circulation, vol. 112, no. 12, pp. e154-e235, 205.

[24] X. L. Wang, J. Y. Mao, and Y. Z. Hou, “TCM clinical evaluating method of disease-syndrome, system segment and multidimensional index," Chinese Journal of Integrative Traditional and Western Medicine, vol. 2, pp. 270-273, 2013. 


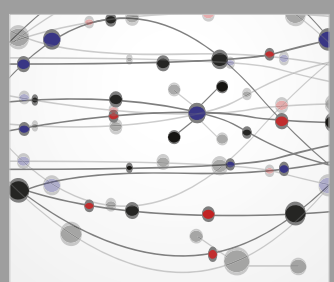

The Scientific World Journal
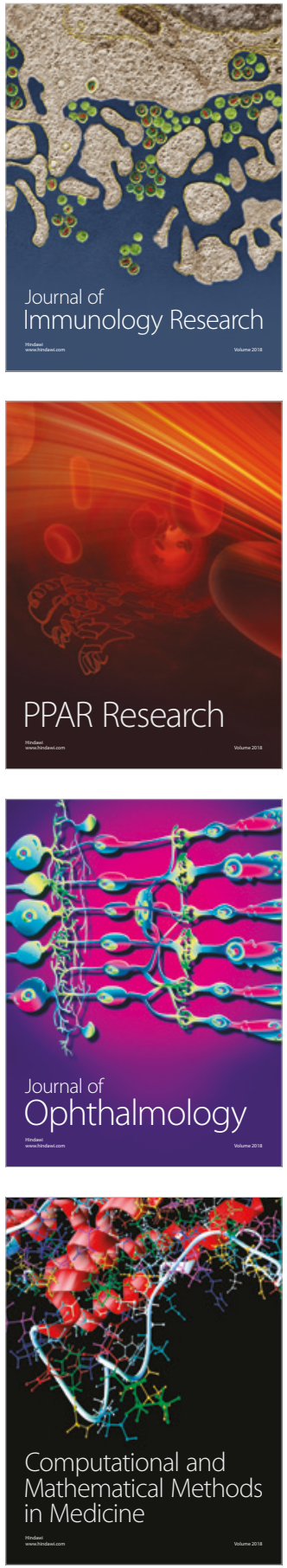

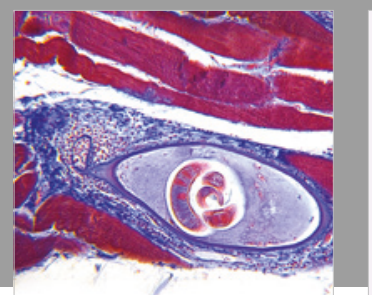

Gastroenterology Research and Practice

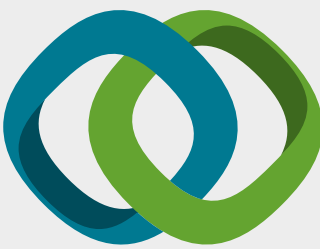

\section{Hindawi}

Submit your manuscripts at

www.hindawi.com
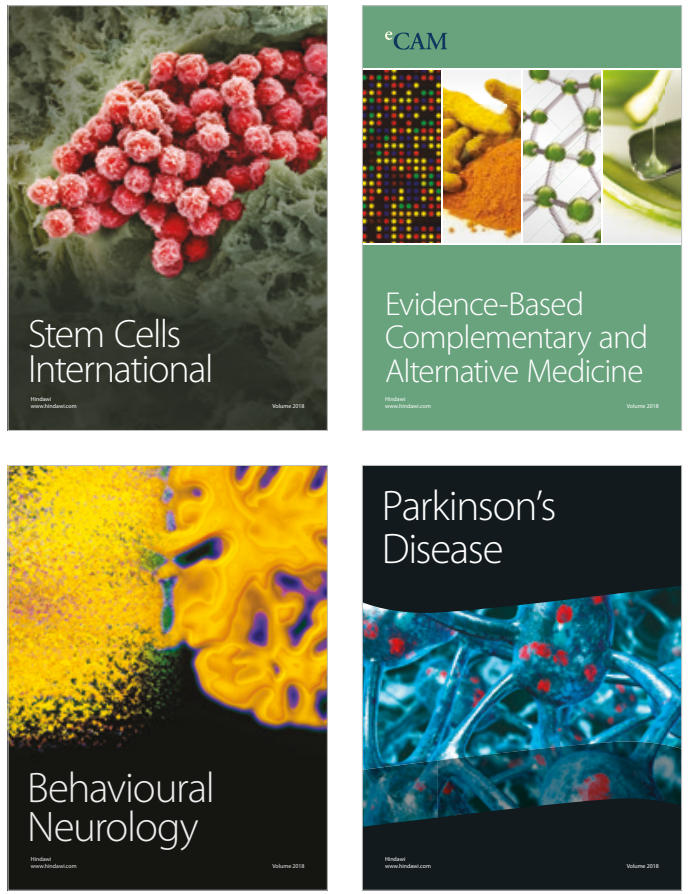

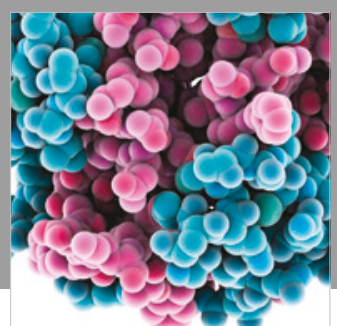

ournal of

Diabetes Research

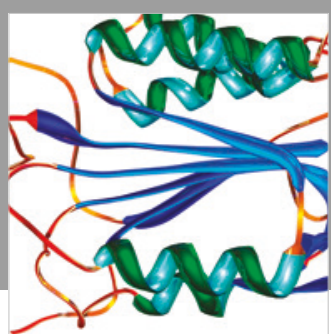

Disease Markers
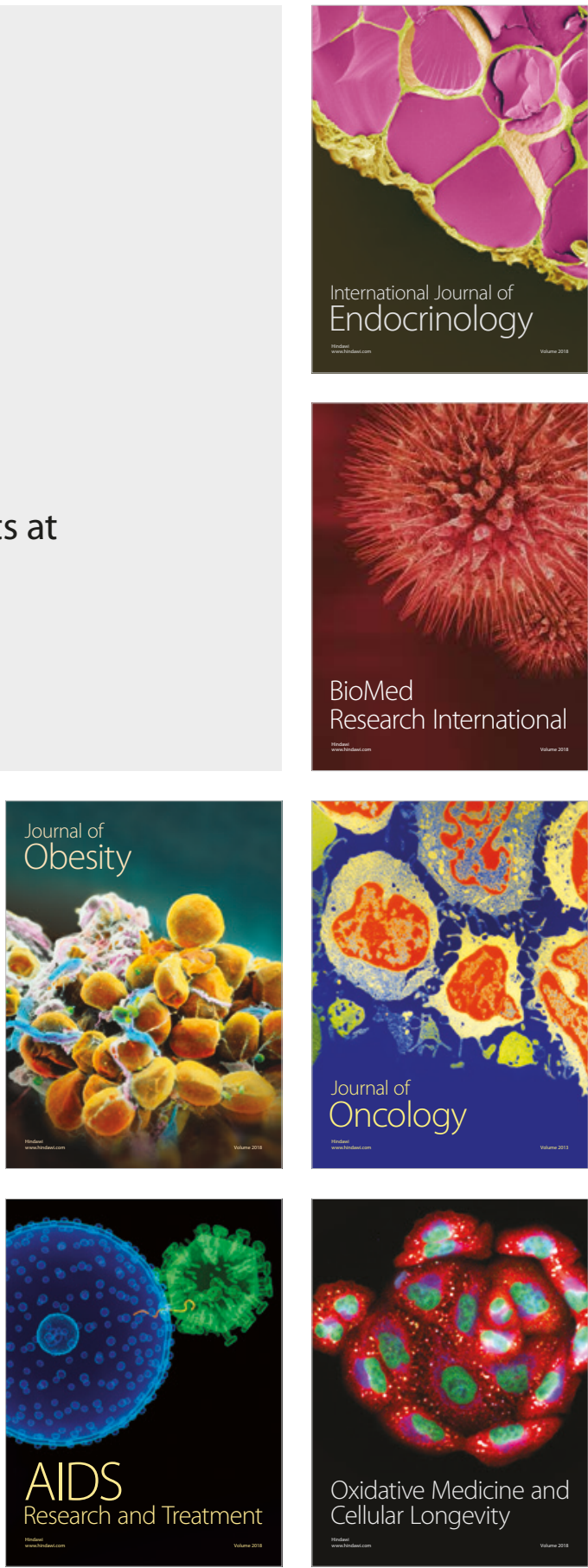